\title{
Scaling Properties and Asymptotic Spectra of Finite Models of Phase Transitions as They Approach Macroscopic Limits
}

\author{
D. J. Rowe and P. S. Turner \\ Department of Physics, University of Toronto, Toronto, Ontario M5S 1A7, Canada \\ G. Rosensteel \\ Department of Physics, Tulane University, New Orleans, Louisiana 70118, USA
}

(Received 3 August 2004; published 30 November 2004)

\begin{abstract}
The asymptotic spectra and scaling properties of a mixed-symmetry Hamiltonian, which exhibits a second-order phase transition in its macroscopic limit, are examined for a system of $N$ interacting bosons. A second interacting boson-model Hamiltonian, which exhibits a first-order phase transition, is also considered. The latter shows many parallel characteristics and some notable differences, leaving it open to question as to the nature of its asymptotic critical-point properties.
\end{abstract}

DOI: $10.1103 /$ PhysRevLett.93.232502

Model studies have contributed enormously to understanding phase transitions and critical phenomena. This Letter reports an attempt to develop a strategy for inferring the large- $N$ behavior of a finite $N$-particle model of a phase transition. Because of the enormous impact of scaling laws on the theory of critical phenomena [1], we seek to determine if the spectral properties of a model have well-defined asymptotic limits with $N$-dependent scale factors. We focus on structural phase transitions in the zero temperature thermodynamic limit [2] of algebraic models with Hamiltonians

$$
\hat{H}_{12}(\alpha)=(1-\alpha) \hat{H}_{1}+\alpha \hat{H}_{2},
$$

such that, when $\alpha=0$ or 1 , the system is in a phase characteristic of the symmetry of $\hat{H}_{1}$ or $\hat{H}_{2}$, respectively.

Figure 1 shows the energy-level spectrum [3] for an interacting boson-model (IBM) Hamiltonian [4]. The IBM comprises $N$ two-state bosons: an $L=0$ (s-boson) state and a higher-energy $L=2(d$-boson) state with five orientations (having a $z$ component of angular momentum $m=0, \pm 1, \pm 2)$. When the bosons are noninteracting, they form a spherically symmetric s-boson condensate. As an interaction is turned on, a point is reached at which a nonspherical phase begins to develop.

The Hilbert space of $N$-boson states carries an irreducible representation of the group $\mathrm{U}(6)$ of unitary transformations of the six boson creation operators $\left\{s^{\dagger}, d_{m}^{\dagger}\right\}$. The IBM is known [4] to be exactly solvable for any $N$ in three special cases corresponding to rotationally invariant Hamiltonians $\hat{H}_{1}, \hat{H}_{2}$, and $\hat{H}_{3}$, which, to within terms in subgroup Casimir invariants, are also invariant under respective $\mathrm{U}(5), \mathrm{O}(6)$, and $\mathrm{SU}(3)$ subgroups of $\mathrm{U}(6)$. For intermediate situations, the model can be solved numerically.

The results of Fig. 1 are for an $\mathrm{SO}(5)$-invariant IBM Hamiltonian $\hat{H}_{12}(\alpha)$ of the form (1) with $\hat{H}_{1}=\hat{n}=2 \hat{S}_{0}^{d}$ and $\hat{H}_{2}=\frac{1}{N} \hat{S}_{+} \hat{S}_{-}$, where $\hat{n}$ is the $\mathrm{U}(5)$-invariant $d$-boson number operator and $\hat{S}_{ \pm}$are elements of an O(6)-invariant
PACS numbers: 21.60.Fw, 21.60.Ev, 68.35.Rh

$\mathrm{SU}(1,1)$ algebra. The latter is spanned by a subset of elements,

$$
\hat{S}_{ \pm}=\hat{S}_{ \pm}^{d}-\hat{S}_{ \pm}^{s}, \quad \hat{S}_{0}=\hat{S}_{0}^{d}+\hat{S}_{0}^{s},
$$

of an $\mathrm{SU}(1,1)^{s} \oplus \mathrm{SU}(1,1)^{d}$ spectrum generating algebra with commutation relations

$$
\begin{array}{ll}
{\left[\hat{S}_{0}^{s}, \hat{S}_{ \pm}^{s}\right]= \pm \hat{S}_{ \pm}^{s}, \quad\left[\hat{S}_{-}^{s}, \hat{S}_{+}^{s}\right]=2 \hat{S}_{0}^{s},} \\
{\left[\hat{S}_{0}^{d}, \hat{S}_{ \pm}^{d}\right]= \pm \hat{S}_{ \pm}^{d}, \quad\left[\hat{S}_{-}^{d}, \hat{S}_{+}^{d}\right]=2 \hat{S}_{0}^{d} .}
\end{array}
$$

For small values of $\alpha$, a large- $N$ limit is obtained by assuming the number $n$ of $d$ bosons in low-energy states of the model remains small compared to $N$; then $\hat{S}_{0}^{s}$ and $\hat{S}_{ \pm}^{s}$ can be replaced by $N / 2$ and

$$
\begin{aligned}
\hat{H}_{12}(\alpha) & \rightarrow \hat{H}_{1}^{\mathrm{eff}}(\alpha)=(2-3 \alpha) S_{0}^{d}-\frac{1}{2}\left(\hat{S}_{+}^{d}+\hat{S}_{-}^{d}\right) \\
& =2 \sqrt{(1-2 \alpha)(1-\alpha)} \hat{S}_{0}^{\prime}(\alpha),
\end{aligned}
$$

where

$$
\hat{S}_{0}^{\prime}(\alpha)=\cosh \varphi \hat{S}_{0}^{d}-\frac{1}{2} \sinh \varphi\left(\hat{S}_{+}^{d}+\hat{S}_{-}^{d}\right)
$$

has the same spectrum as $\hat{S}_{0}^{d}$. The asymptotic energy

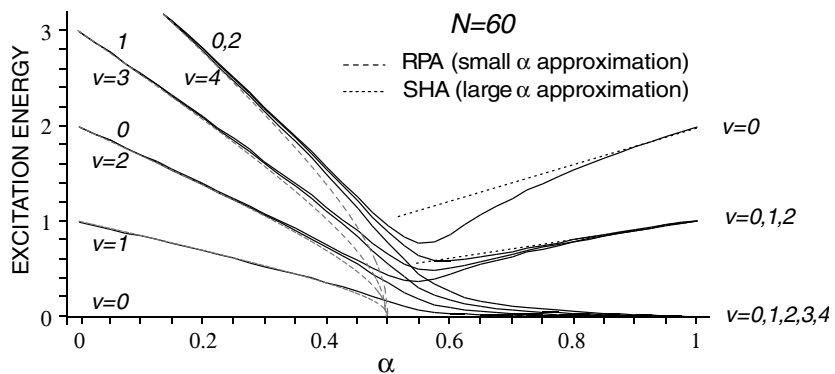

FIG. 1. Excitation energies of the Hamiltonian $\hat{H}_{12}(\alpha)$ of Eq. (1) for a system of 60 bosons as a function of $\alpha$. Each energy level is an $\mathrm{SO}(5)$ multiplet of states and is labeled by an $\mathrm{SO}(5)$ angular momentum $v$ known as seniority. 
spectrum is then given by $E_{n v}(\alpha)=n \sqrt{(1-2 \alpha)(1-\alpha)}$. Thus, to within $\alpha$-dependent renormalization factors, the properties of the above model remain those of its $\alpha=0$ limit for a subset of low-energy states and a range of values of $\alpha$ for which $n$ remains small compared to $N$.

This small- $\alpha$ large- $N$ approximation is equivalent to the random phase approximation (RPA) of Ref. [3] and becomes precise as $N \rightarrow \infty$ for $\alpha$ less than the critical value $\alpha_{c}=0.5$ at which its excitation energies collapse to zero and the number of $d$-bosons in the ground state is predicted to become macroscopic. Thus, the RPA fails for finite $N$ as $\alpha$ approaches 0.5 , as shown in Fig. 1

For $\alpha=1$, the spectrum of the operator $\hat{S}_{+} \hat{S}_{-}$, on the $N$-boson Hilbert space, becomes that of a harmonic oscillator (HO) as $N \rightarrow \infty$. Thus, $\hat{S}_{+} \hat{S}_{-} \rightarrow N A^{\dagger} A$, where $A^{\dagger}$ and $A$ are $\mathrm{HO}$ raising and lowering operators [3]. Moreover, in this $\mathrm{HO}$ representation, the $d$-boson number operator $\hat{n} \rightarrow \frac{1}{2} \sqrt{N}\left(A^{\dagger}+A\right)$ and

$$
\hat{H}_{12}(\alpha) \rightarrow \hat{H}_{2}^{\text {eff }}=\alpha\left(A^{\dagger}+z\right)(A+z)+\text { const, }
$$

where $z=\frac{1}{2} \sqrt{N}(1-\alpha) / \alpha$. This result shows that, whereas the eigenstates are HO states at $\alpha=1$, they are shifted HO (i.e., Glauber coherent) states for $\alpha<1.0$. The corresponding asymptotic energy spectrum, given by $E_{n v}(\alpha)=n \alpha$, is shown in Fig. 1. To within an $\alpha$-dependent renormalization factor, the properties of the model are now predicted to remain those of its $\alpha=$ 1 limit for a subset of low-energy states for which the shifted harmonic approximation (SHA) is valid. Again the SHA is precise for $\alpha>0.5$ as $N \rightarrow \infty$ but fails for finite $N$ as $\alpha$ approaches 0.5 from above.

The apparent persistence of symmetries, associated in this case with the $\mathrm{U}(5)$ and $\mathrm{O}(6)$ symmetries of the $\alpha=0$ and $\alpha=1$ limits, has been dubbed quasidynamical symmetry. Such quasidynamical symmetry has been widely observed [5] and appears to be a common property of systems with competing symmetries.

The above results illustrate how, from contraction limits of the algebraic structure of a model, it is possible to get asymptotically precise results for a model outside of a transition zone which shrinks to a singular critical point as $N \rightarrow \infty$. A recent observation [6] that critical points can sometimes be associated with exactly solvable effective Hamiltonians suggests that a system may even have scalable asymptotic spectra at a singular critical point.

Figure 2(a) shows the low-energy levels of the above model at the critical point $\alpha=0.5$ in units of the energy of the first excited state for a range of $N$. The remarkable result is that the energy levels retain the same order for $N \geq 5$ and appear to rapidly approach constant asymptotic values with increasing $N$. This result suggests that the spectrum of energy levels at the critical point is given accurately for $N \geqslant 50$ by an effective Hamiltonian

$$
\hat{H}_{12}^{\text {eff }}(0.5)=k(N) \hat{H}_{0},
$$

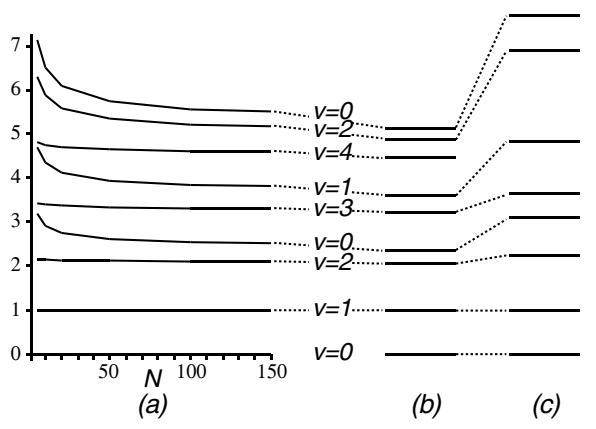

FIG. 2. Excitation energies for levels of $v \leq 4$ in units of the lowest $v=1$ excitation energy for $\alpha=0.5$ : (a) for the Hamiltonian $\hat{H}_{12}(\alpha)$ of Eq. (1) and a range of $N$ values; (b) for the collective model Hamiltonian $\hat{H}_{\mathrm{CM}}(\alpha)$ of Eq. (10); and (c) for an infinite square-well potential (from Ref. [6]).

where $\hat{H}_{0}$ is the $N_{0}$-boson Hamiltonian $\hat{H}_{12}(0.5)$ for some large value of $N_{0}$, and $k(N)$ is an $N$-dependent scale factor. Numerical calculation shows that the energy scale is given quite accurately, for $N$ in the 5-200 range, by

$$
k(N) \approx k_{1} N^{-0.3} \text {. }
$$

The above results are readily understood by means of a related collective model Hamiltonian

$$
\hat{H}_{\mathrm{CM}}(\alpha)=-\frac{\nabla^{2}}{2 M}+V_{\alpha}(\beta),
$$

where $\nabla^{2}$ is the Laplacian on a five-dimensional Euclidean space and $\beta^{2}$ is the squared length $\beta^{2}=q$. $q$ of a vector $q$ in this space. With $M=2 N, V_{\alpha}(\beta)$ is the classical energy function defined [3,7,8] for $N$ bosons with Hamiltonian $\hat{H}_{12}(\alpha)$ by

$$
V_{\alpha}(\beta)=\left\langle q\left|\hat{H}_{12}(\alpha)\right| q\right\rangle=\frac{1}{2} M\left[(1-2 \alpha) \beta^{2}+\alpha \beta^{4}\right],
$$

where $|q\rangle=\frac{1}{\sqrt{N !}}\left(B^{\dagger}\right)^{N}|0\rangle$ is a $\mathrm{U}(6)$ coherent state with $B^{\dagger}=\sqrt{1-\beta^{2}} s^{\dagger}+\sum_{m} q^{m} d_{m}^{\dagger}$. This potential has a minimum at $\beta=0$ for $\alpha \leq 0.5$, at $\beta>0$ for $\alpha \geq 0.5$, and exhibits a second-order phase transition [8] from a spherical to a deformed $(\beta>0)$ phase at $\alpha=0.5$ [9].

At the critical point, $\hat{H}_{\mathrm{CM}}(0.5)$ takes the form

$$
\hat{H}_{\mathrm{CM}}(0.5)=\frac{1}{2} M^{-1 / 3}\left[-\tilde{\nabla}^{2}+\tilde{\beta}^{4}\right] \text {, }
$$

where $\tilde{\beta}=M^{1 / 3} \beta$. Thus it has a constant spectrum with an $M^{-1 / 3}$ scale factor. This spectrum, previously derived by Arias et al. [10] and shown in Fig. 2(b), is remarkably similar to the asymptotic spectrum suggested by the IBM results of Fig. 2(a). The $M^{-1 / 3}$ scale factor is also close to that of Eq. (9) with $M=2 N$.

In his $\mathrm{E}(5)$ model, Iachello [6] employs an infinite square well in place of the $\beta^{4}$ potential of Eq. (12). It has an asymptotic spectrum, shown in Fig. 2(c), that agrees well with that of the IBM results of Fig. 2(a) for $N \lesssim 5$ (cf. Ref. [11]). A sequence of $\beta^{2 n}$ potentials was 
considered by Bonatsos et al. [12] and was found to reproduce the $\mathrm{E}(5)$ model results for large values of $N$. Other proposals of numerically solvable approximations for the critical-point spectrum are discussed, for example, in Refs. $[8,13]$.

The utility of finite models of phase transitions with predictable macroscopic limits prompts one to ask what is special about such models. As shown above, quasidynamical symmetry can often be understood in terms of contraction limits of the dynamical symmetries associated with the distinct phases of a model. In such cases, when there are precise $1 / N \rightarrow 0$ limits, it may be possible to predict both the asymptotic spectra and the scale factors for the domains in which the asymptotic limits apply. A more difficult problem is to determine the asymptotic properties of a model at its critical points.

Models with flat (Euclidean) configuration spaces and Hamiltonians $\hat{H}=-\nabla^{2} /(2 M)+V_{\alpha}(\beta)$ are among the most predictable. If such a model exhibits a second-order phase transition and has a potential $V_{\alpha_{c}}(\beta)=k \beta^{n}$ at the critical point, then, if $k$ is independent of the mass parameter, the model spectrum is scale invariant and has an $M^{-n /(n+1)}$ scale factor. More generally, if $V_{\alpha_{c}}$ has a minimum at $\beta=0$, then the spectrum of $\hat{H}$ will have an asymptotic limit with a scale factor given by the leading term in its polynomial expansion. However, such an asymptotic behavior may become apparent only at large values of $N$. For a first-order phase transition, the potential $V_{\alpha_{c}}$ should have two equal-energy minima, one at $\beta=0$ and another at some $\beta_{0}>0$ with a potential barrier between them. For large values of the mass, when the barrier-penetration effects become negligible, it will then be expected that the spectrum will comprise both spherical states localized about $\beta=0$ and deformed states localized about $\beta=\beta_{0}$, as well as combinations of the two; the lowest-energy spherical and deformed states simply cross at the critical point. One can then expect an asymptotic spectrum, albeit with multiple scaling factors which apply to different components of the spectrum.

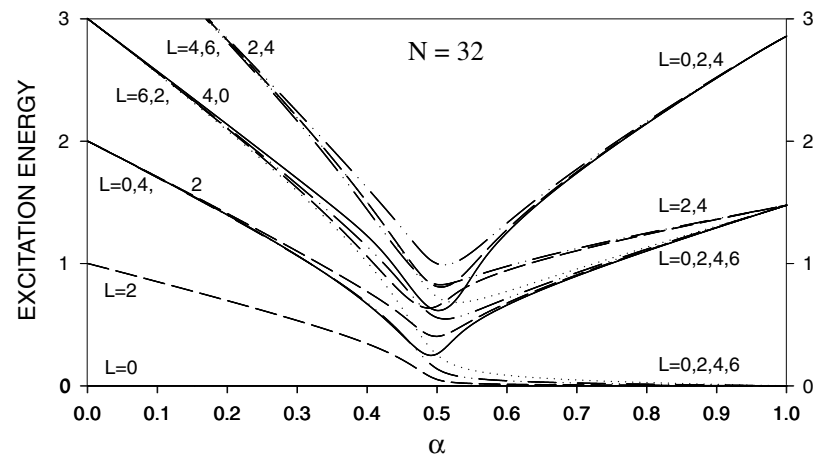

FIG. 3. Excitation energies of the Hamiltonian $\hat{H}_{13}(\alpha)$ of Eq. (13) for a system of 32 bosons as a function of $\alpha$.
The following are preliminary results [14] for an IBM first-order phase transition [15] with Hamiltonian

$$
\hat{H}_{13}(\alpha)=(1-\alpha) \hat{H}_{1}+\alpha \hat{H}_{3},
$$

where $\hat{H}_{1}=\hat{n}$, as before, and $\hat{H}_{3}$ is proportional to the $\mathrm{SU}(3)$ Casimir operator. Figure 3 shows the energy levels for $\hat{H}_{13}$ as a function of $\alpha$ for $N=32$. Quasidynamical $\mathrm{U}(5)$ and $\mathrm{SU}(3)$ symmetries are now as persistent as they were for the $\mathrm{U}(5)$ and $\mathrm{O}(6)$ phases of the first model considered. Again, the transition zone between the two phases narrows as $N$ increases. This is exhibited dramatically in Fig. 4, which shows the ratio of electric quadrupole transition rates between the lowest $L=4,2$, and 0 states. Figure 4 shows that, as $N$ becomes large, the ratios of transition rates remain close to their $\alpha=0$ or $\alpha=1$ values for all $\alpha$ outside of a transition zone that become increasingly narrow as $N$ increases. A feature particularly evident for $0.6<\alpha<1.0$ in Fig. 3 is the appearance of near-linear splittings of some multiplets that do not break their quasidynamical symmetries.

Figure 5 shows critical-point spectra for $\hat{H}_{13}$ in units of the energy of the lowest $L=2$ excitation energy for $N=$ 10 and 60 . The band structures of the spectra are qualitatively similar, and it is conceivable that they could be progressing towards large- $N$ asymptotic limits with one or more scaling factors. However, more results are required before it is possible to draw this conclusion. To see if the ground-state band follows a recognizable scaling law, we have calculated the energies of the first excited state up to $N=150$. The results are shown on a log-log plot in Fig. 6. The figure shows that the excitation energy of this state scales approximately with $N^{-0.43}$ for $N \approx 10$ and as $N^{-1}$ for $N \approx 150$. However, the figure gives no indication of an asymptotic exponent.

One can attempt to understand these results by considering the corresponding collective model as for the boson model of a second-order phase transition. The classical potential energy $V_{\alpha}(\beta, \gamma)=\left\langle q\left|\hat{H}_{13}(\alpha)\right| q\right\rangle$ is now

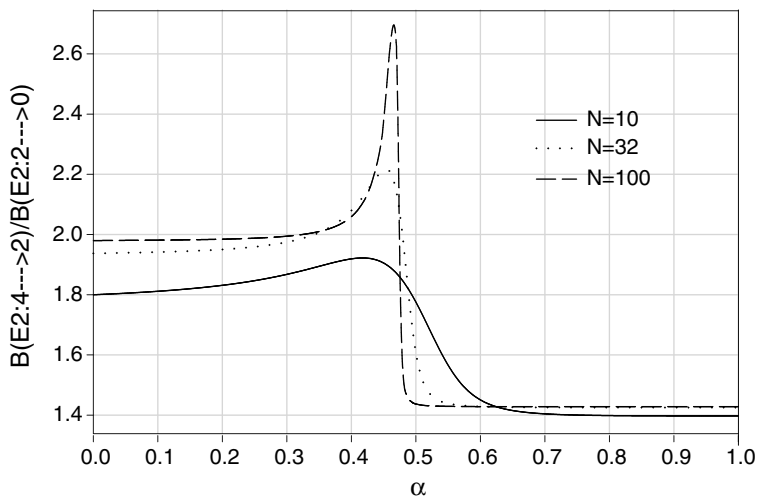

FIG. 4. The ratio $B(E 2: 4 \rightarrow 2) / B(E 2: 2 \rightarrow 0)$ for $N=10,32$, and 100. 


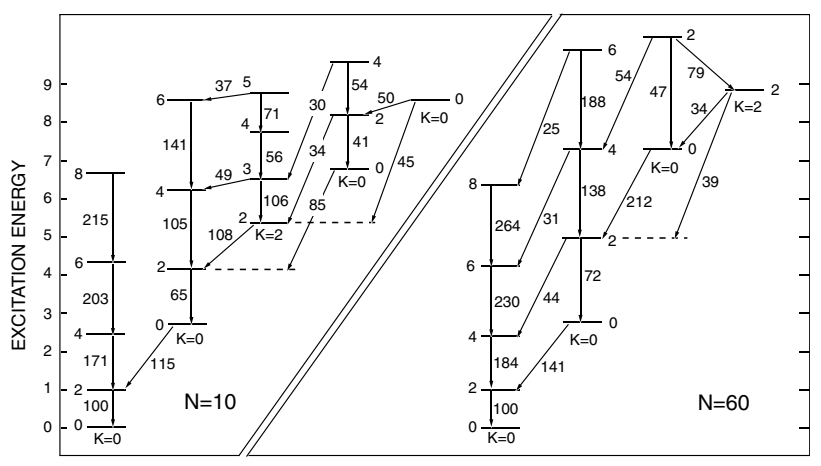

FIG. 5. Excitation energies for some low-lying states of the Hamiltonian $\hat{H}_{13}$ at the critical point for $N=10$ and 60 , in units of the excitation energy of the first excited state.

$$
\begin{aligned}
V_{\alpha}(\beta, \gamma)= & N\left[1-\left(2-\frac{25}{16 N}\right) \alpha\right] \beta^{2}+(N-1) \alpha\left[\frac{7}{16} \beta^{4}\right. \\
& \left.-\sqrt{\left(1-\beta^{2}\right) / 2} \beta^{3} \cos 3 \gamma\right]
\end{aligned}
$$

where $\beta, \gamma$, and $\Omega \in \mathrm{SO}(3)$ are spherical polar coordinates for the quadrupole moments defined by

$$
\begin{aligned}
q_{\mu}= & \beta \cos \gamma \mathcal{D}_{0 \mu}^{2}(\Omega)+\frac{1}{\sqrt{2}} \beta \sin \gamma\left[\mathcal{D}_{2 \mu}^{2}(\Omega)\right. \\
& \left.+\mathcal{D}_{-2, \mu}^{2}(\Omega)\right] .
\end{aligned}
$$

This potential has two minima for some values of $\alpha$ : at $\beta=0$ and at $(\beta \approx 0.5, \gamma=0)$. Thus, at a critical $\alpha$ the classical equilibrium configuration jumps discontinuously from a spherical $\beta=0$ shape to an axially symmetric $\beta \approx 0.5$ shape in the manner of a first-order phase transition. However, it turns out that the potential barrier between the two minima is extremely small. In this situation, the collective model would lead one to expect that the asymptotic properties of a first-order phase transition region would be approached only for this model at extremely large values of $N$ consistent with our findings.

Iachello [15] has predicted an approximate criticalpoint spectrum for $\hat{H}_{13}(\alpha)$ corresponding to an exactly solvable effective Hamiltonian with a so-called X(5) model which dispenses with the barrier and approximates the potential (14) with an infinite square well in the $\beta$ variable plus a harmonic oscillator potential in $\gamma$. The $\mathrm{X}(5)$ model predicts a critical-point spectrum that is qualitatively similar to our boson-model results for small values of $N$. This suggests the possibility that there could be a range of relatively small values of $N$, over which the potential (14) is approximately scale invariant.

The above results suggest that, because of the tiny barrier between the two-degenerate minima at the critical point, the asymptotic scaling properties of the boson model of a first-order phase transition considered will be observable only at extremely large values of the boson

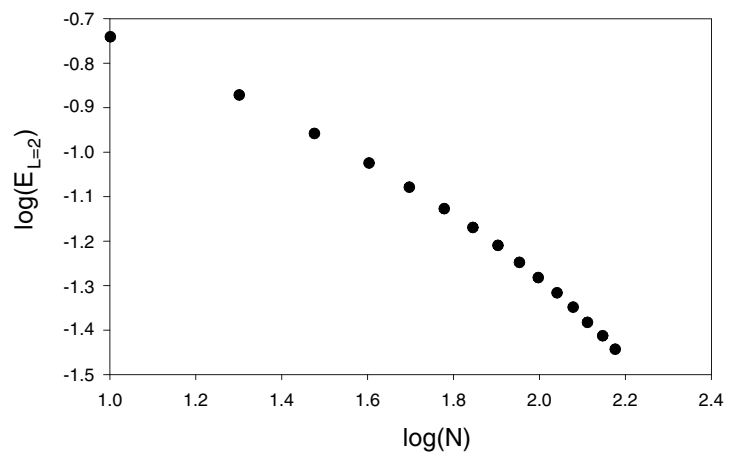

FIG. 6. A log-log plot of the excitation energies of the first excited state of $\hat{H}_{13}$ at the critical point as a function of $N$.

number. Thus, it will be of considerable interest to explore other models for which this is not the case.

[1] N. Goldenfeld, Lectures on Phase Transtions and the Renormalization Group, Frontiers of Physics Vol. 85 (Perseus Books, Reading, 1992).

[2] P. Cejnar, S. Heinze, and J. Jolie, Phys. Rev. C 68, 034326 (2003); F. Iachello, in Quantum Phase Transitions in Nuclei and Other Systems, Proceedings of the International School of Physics "Enrico Fermi," Course CLIII, edited by A. Molinari, L. Riccati, W. M. Alberico, and M. Morando (IOS Press, Amsterdam, 2003).

[3] D. J. Rowe, Nucl. Phys. A745, 47 (2004).

[4] A. Arima and F. Iachello, Ann. Phys. (N.Y.) 99, 253 (1976); 111, 201 (1978); O. Scholten, A. Arima, and F. Iachello, Ann. Phys. (N.Y.) 115, 325 (1978).

[5] D. J. Rowe, in Computational and Group Theoretical Methods in Nuclear Physics, edited by O. Castańos, J. Escher, J. Hirsch, S. Pittel, and G. Stoicheva (World Scientific, Singapore, 2004).

[6] F. Iachello, Phys. Rev. Lett. 85, 3580 (2000).

[7] R. Gilmore, J. Math. Phys. (N.Y.) 20, 891 (1979).

[8] A. E. L. Dieperink, O. Scholten, and F. Iachello, Phys. Rev. Lett. 44, 1747 (1980); O. S. Roosmalen and A. E. L. Dieperink, Phys. Lett. 100B, 299 (1981).

[9] D. J. Rowe and P. S. Turner (to be published).

[10] J. M. Arias, C. E. Alonso, A. Vitturi, J. E. García-Ramos, J. Dukelsky, and A. Frank, Phys. Rev. C 68, 041302 (2003).

[11] A. Leviatan and J. N. Ginocchio, Phys. Rev. Lett. 90, 212501 (2003).

[12] D. Bonatsos, D. Lenis, N. Minkov, P. P. Raychev, and P. A. Terziev, Phys. Rev. C 69, 044316 (2004); D. Bonatsos, D. Lenis, N. Minkov, D. Petrellis, P. P. Raychev, and P. A. Terziev, Phys. Lett. B 584, 40 (2004).

[13] P. Cejnar and J. Jolie, Phys. Rev. E 61, 6237 (2000); M. A. Caprio, Phys. Rev. C 65, 031304(R) (2202); L. Fortunato and A. Vitturi, J. Phys. G 30, 627 (2004).

[14] G. Rosensteel and D. J. Rowe (to be published).

[15] F. Iachello, Phys. Rev. Lett. 87, 052502 (2001). 